ISSN 0103-9954

\title{
ACÚMULO DE BIOMASSA E NUTRIENTES DE DUAS LEGUMINOSAS ARBÓREAS INTRODUZIDAS EM SISTEMA DE POUSIO NA AMAZÔNIA
}

\author{
BIOMASS AND NUTRIENT ACCUMULATION OF TWO LEGUMINOUS TREES IN AN \\ IMPROVED FALLOW IN AMAZON RAIN FOREST
}

\author{
Lívia Gabrig Turbay Rangel-Vasconcelos ${ }^{1}$ Osvaldo Ryohei Kato ${ }^{2}$ Steel Silva Vasconcelos ${ }^{3}$ \\ Francisco de Assis Oliveira ${ }^{4}$
}

\begin{abstract}
RESUMO
A vegetação de pousio é um dos principais componentes dos sistemas agroflorestais sequenciais de derrubada e queima, praticados tradicionalmente pela agricultura familiar na Amazônia. A remoção progressiva de nutrientes do solo por essa prática implica reduções contínuas nos estoques de carbono e nutrientes causando declínio da produtividade do solo, perda da capacidade de regeneração e diminuição da diversidade de espécies da vegetação. O melhoramento de pousio com espécies leguminosas fixadoras do nitrogênio atmosférico pode contribuir para uma maior produção de biomassa e acúmulo de nutrientes em comparação com a vegetação espontânea, atendendo à demanda nutricional das culturas subsequentes, podendo ser considerada como uma tecnologia de produção sustentável. Esse trabalho avaliou o efeito da adubação fosfatada de baixa solubilidade no acúmulo de biomassa e nutrientes da parte aérea de leguminosas arbóreas utilizadas em melhoramento de vegetação de pousio. O experimento foi conduzido por 23 meses, em um sistema agroflorestal de "corte e trituração" em Marapanim, Pará. Foram utilizadas as espécies tachi-branco - Sclerolobium paniculatum Vogel e ingá - Inga edulis Mart e foram estimados biomassa, teor e estoque de nutrientes dos compartimentos folha, galho e tronco. Houve acúmulo de biomassa, estoques de $\mathrm{P}, \mathrm{Ca}$ e $\mathrm{Mg}$ nas folhas, estoque de $\mathrm{P}$ nos galhos e o teor de cálcio nas folhas das leguminosas é limitado por fósforo, segundo os resultados encontrados. A espécie Inga edulis apresentou maiores teores de potássio, cálcio e magnésio nos compartimentos folha, galho e tronco, enquanto que a espécie Sclerolobium paniculatum apresentou maiores acúmulos de biomassa e estoques de nitrogênio, fósforo, potássio, cálcio e magnésio nos compartimentos folha, galho e tronco.
\end{abstract}

Palavras-chave: Sclerolobium paniculatum; Inga edulis; corte-e-trituração; capoeira.

\begin{abstract}
The fallow vegetation is the main component of sequential agroforestry systems in Amazon rain forest.The progressive removal of soil nutrient by slash/mulch agriculture involves continuing losses in carbon and nutrient stocks, leading to decline in soil productivity, low regeneration ability and decrease of species diversity of fallow vegetation. The fallow vegetation management by introducing fast growing leguminous trees has the potential to accumulate more biomass and nutrients to serve the nutrient demands of crops and its management has been considered as a sustainable technology production. This study aimed to evaluate the effect of low solubility of phosphorus fertilizer in the biomass and nutrient stocks of leguminous trees used in fallow improvement. The experiment was conducted for 23 months in a sequential agroforestry slash-and-mulch

1 Engenheira Florestal, Dra., Professora Adjunta, Universidade Federal Rural da Amazônia Federal Rural da Amazônia, Caixa Postal 917, CEP 66077-530, Belém (PA), Brasil. liviaturbay@gmail.com

2 Engenheiro Agrônomo, PhD., Pesquisador A, Embrapa Amazônia Oriental, Tv. Dr Enéas Pinheiro, s/n, Bairro Marco, Caixa postal 48, CEP 66095-100, Belém (PA), Brasil. osvaldo.kato@embrapa.br

3 Engenheiro Agrônomo, PhD., Pesquisador A, Embrapa Amazônia Oriental, Tv. Dr Enéas Pinheiro, s/n, Bairro Marco, Caixa postal 48, CEP 66095-100, Belém (PA), Brasil. Steel.vasconcelos@embrapa.br

4 Engenheiro Florestal, Dr., Professor Associado III do Instituto de Ciências Agrárias, Universidade Federal Rural da Amazônia, Caixa Postal 917, CEP 66077-530, Belém (PA), Brasil. fdeassis@gmail.com
\end{abstract}

Recebido para publicação em 30/04/2013 e aceito em 30/10/2014

Ci. Fl., v. 26, n. 3, jul.-set., 2016 
system in eastern Amazon forest. The species selected were tachi-branco - Sclerolobium paniculatum Vogel and inga - Inga edulis Mart. Biomass and nutrient stocks and nutrient concentrations of leaf, branch and trunk were estimated. Phosphorus fertilization had a positive effect on calcium concentration in the leaves and stocks of biomass and nutrient contents (phosphorus, calcium and magnesium) in the leaves of legume trees. Inga edulis showed higher levels of potassium, calcium and magnesium concentrations in leaves, while Sclerolobium paniculatuttm showed higher biomass and stocks of nitrogen, phosphorus, potassium, calcium and magnesium in leaf, branch and trunk.

Keywords: Sclerolobium paniculatum; Inga edulis; slash-and-mulch; capoeira.

\section{INTRODUÇÃO}

A vegetação secundária é o componente principal dos sistemas agroflorestais sequenciais, representados principalmente pelos sistemas de itinerante de derrubada e queima adotados pela maioria dos agricultores familiares na Amazônia brasileira. O sistema de derrubada e queima é caracterizado por fases alternadas de pousio período de crescimento da vegetação secundária - e de cultivo agrícola. O manejo da vegetação de pousio é importante para manutenção da produtividade desse sistema, pois, durante este período, o sistema acumula nutrientes para atender à demanda nutricional das culturas agrícolas durante a fase de cultivo.

A remoção progressiva de nutrientes do solo pela agricultura de derrubada e queima implica reduções contínuas dos estoques de carbono e nutrientes (SOMMER, 2000; ZARIN et al., 2005), causando declínio da produtividade do solo (ALMEIDA et al., 2006; DENICH et al., 2005), além da redução da capacidade de regeneração da vegetação e diversidade de espécies (VOCKEL et al., 2000). Esses efeitos negativos são minimizados quando o período de pousio é suficientemente longo (aproximadamente 10 anos), permitindo o restabelecimento da vegetação secundária e dos estoques de matéria orgânica do solo. No entanto, nas últimas décadas tem se observado uma diminuição do período de pousio, sobretudo na Amazônia oriental, em função da intensificação do uso da terra, crescimento populacional e aumento da demanda por produção de alimentos (DENICH et al., 2005).

O pousio melhorado (SANCHEZ, 1999) com leguminosas arbóreas fixadoras de nitrogênio e de rápido crescimento tem sido considerado como tecnologia de produção sustentável para a agricultura familiar na Amazônia (BRIENZA JÚNIOR, 2012), pois tem o potencial de produzir biomassa e acumular nutrientes em níveis superiores ao que a vegetação espontânea poderia atingir durante um determinado período de pousio, promovendo a adição de carbono e nutrientes no sistema, recuperando gradativamente a qualidade do solo (BARRIOS et al., 2004, BASAMBA et al., 2007). Em solos tropicais, notadamente deficientes em fósforo disponível às plantas (VITOUSEK et al., 1986), a adubação fosfatada das espécies utilizadas no melhoramento pode contribuir para um maior acúmulo de biomassa e nutrientes durante o período de pousio (LAWRENCE, 2001, MCGRATH et al., 2001, SANTOS JR et al., 2006). Dessa forma, a introdução de espécies leguminosas associadas à adubação fosfatada de baixa solubilidade pode promover o acúmulo de biomassa e nutrientes que podem influenciar positivamente na produtividade das culturas agrícolas.

Experimentos com fertilização detectam limitação por nutrientes em agroecossistemas (GÜSEWELL, 2004, KOERSELMAN et al., 1996). A adubação fosfatada das leguminosas arbóreas utilizadas em manejo da vegetação de pousio visando ao acúmulo de biomassa é sugerida na literatura (MCGRATH et al., 2001, SANCHEZ, 1999), entretanto, não há relato de estudos sobre o desempenho dessas espécies, submetidas à adubação fosfatada, em acumular biomassa e nutrientes na vegetação de pousio.

O objetivo deste trabalho foi avaliar o efeito da adubação fosfatada sobre o acúmulo de massa seca e de nutrientes em leguminosas arbóreas utilizadas em pousio melhorado, na Amazônia Oriental.

\section{MATERIAL E MÉTODOS}

\section{Descrição da área}

O estudo foi realizado, em uma propriedade de agricultura familiar, na Comunidade São João 
(100’4” S, 47³8’3” W), município de Marapanim que está incluído na Zona Bragantina, uma das áreas mais antigas de colonização na Amazônia, com uso intenso dos recursos naturais.

O clima da região é do tipo “Ami”, segundo a classificação de Köppen, tropical sem ocorrência de inverno estacional, com temperatura média anual de $26^{\circ} \mathrm{C}$ e umidade relativa do ar de 80 a $89 \%$. A precipitação média anual é de $2506 \pm 212 \mathrm{~mm}$ (média \pm desvio padrão), com período de maior precipitação de janeiro a junho e menor precipitação de setembro a novembro.

Os solos da região são classificados como Latossolo amarelo de textura média, derivado da formação Barreiras. A caracterização química e granulométrica do solo da área experimental antes do início dos tratamentos está apresentada na Tabela 1.

\section{Histórico e descrição do experimento}

Em maio de 2006, um fragmento (0,5 ha) de vegetação secundária de oito anos de idade foi triturado com fresador florestal do tipo AHWI FM 600. Um mês após a trituração, foi plantada mandioca - Manihot esculenta (cultivar cearense) no espaçamento $1 \mathrm{~m} \mathrm{x} 1 \mathrm{~m}$. Em junho de 2007, foram plantadas mudas das leguminosas arbóreas Inga edulis Mart. e Sclerolobium paniculatum Vogel entre as linhas de Manihot esculenta. As leguminosas foram plantadas alternadamente e espaçadas em 2 $\mathrm{m} \times 2 \mathrm{~m}$. A adubação utilizada foi o fosfato natural parcialmente acidulado (Arad), na dosagem de 200 g por cova das leguminosas introduzidas no sistema (165 kg de $\left.\mathrm{P}_{2} \mathrm{O}_{5} \mathrm{ha}^{-1}\right)$.

A escolha das espécies foi baseada em estudo realizado anteriormente na mesma região deste estudo (BRIENZA, 1999). Em outubro de 2007 foi realizada manualmente a colheita da mandioca e a roçagem da área da projeção da copa das leguminosas introduzidas ao sistema.

$\mathrm{O}$ experimento foi delineado em blocos ao acaso com quatro repetições e dois tratamentos: (1) (1) plantio de Inga edulis e Sclerolobium paniculatum; (2) plantio de Inga edulis e Sclerolobium paniculatum + adubação fosfatada, totalizando oito unidades amostrais de $10 \mathrm{~m} \mathrm{x} 12$ $\mathrm{m}$. Os tratamentos receberam manejo, como capina durante o cultivo da mandioca, coroamento das leguminosas, conforme os critérios do agricultor.

Em maio de 2009, após 23 meses de pousio, foi realizada a estimativa da biomassa dos
Leguminosas plantadas

\section{Crescimento das leguminosas}

Foram realizadas medições mensais de altura, diâmetro do coleto e diâmetro a altura do peito em todos os indivíduos de Inga edulis e Sclerolobium paniculatum na área útil $(6 \mathrm{~m} \times 8 \mathrm{~m})$ de cada parcela. A altura foi mensurada com vara métrica entre setembro de 2007 e abril de 2009. O diâmetro do coleto e o diâmetro a altura do peito foram mensurados com paquímetro digital de maio de 2008 a abril de 2009 e setembro de 2008 a abril de 2009 , respectivamente.

A altura inicial das plantas de Inga edulis e Sclerolobium paniculatum foi aproximadamente $34,6 \mathrm{~cm}(\mathrm{n}=40)$ e $28,4 \mathrm{~cm}(\mathrm{n}=40)$, respectivamente. A mortalidade das mudas de $I$. edulis em janeiro de 2008 (aos sete meses após plantio) foi de 4\% ( \pm 4) e $13 \%( \pm 8)$ nos tratamentos não adubados e adubados respectivamente, e em maio de 2009 (aos 22 meses após o plantio) foi de $13 \%( \pm 8)$ e $8 \%$ $( \pm 8)$ nos tratamentos não adubados e adubados, respectivamente. A mortalidade das mudas de $S$. paniculatum em janeiro de 2008 foi de $25 \%( \pm 8)$ para o tratamento não adubado e $25 \%( \pm 8)$ para o tratamento adubado e em maio de 2009 foi de $13 \%$ $( \pm 4)$ e $17 \%( \pm 5)$ para os tratamentos adubados e não adubados respectivamente.

\section{Amostragem biomassa da parte aérea}

Em maio de 2009, 23 meses após o de plantio das leguminosas, foram selecionados nove indivíduos dentro da área útil de cada parcela para determinação da biomassa seca da parte aérea, pelo método direto (destrutivo). Este método consistiu no corte e na pesagem separadamente dos compartimentos tronco, galho e folha dos indivíduos selecionados. Subamostras, com aproximadamente $0,5 \mathrm{~kg}$, foram pesadas no campo e, posteriormente, foram secas em estufa de circulação forçada de ar a $65^{\circ} \mathrm{C}$ até atingir peso constante. A massa seca das amostras foi calculada a partir da umidade das subamostras, obtida pela massa úmida e seca das subamostras.

Em seguida, as subamostras foram moídas, em moinho tipo Willey, para a determinação das concentrações de carbono e nutrientes (nitrogênio, fósforo, potássio, cálcio e magnésio). 
TABELA 1: Propriedades química e granulométrica do solo da área experimental em junho de 2007, município de Marapanim, PA.

TABLE 1: Chemical and particle size properties of the experimental area in June 2007, in the municipality of Marapanim, PA state.

\begin{tabular}{cccccccccccccccc}
\hline Profundidade & $\mathrm{pH}_{\mathrm{H} 20}$ & $\mathrm{~N}$ & $\mathrm{MO}$ & $\mathrm{P}$ & $\mathrm{K}$ & $\mathrm{Na}$ & $\mathrm{Ca}$ & $\mathrm{Mg}$ & $\mathrm{Al}$ & $\mathrm{H}+\mathrm{Al}$ & $\begin{array}{c}\text { Areia } \\
\text { Grossa }\end{array}$ & Areia fina & $\begin{array}{c}\text { Silte } \\
\begin{array}{c}\text { Argila } \\
\text { total }\end{array}\end{array}$ \\
\hline $\mathrm{Cm}$ & & $\%$ & $\mathrm{~g} \mathrm{~kg}^{-1}$ & \multicolumn{1}{c}{$\mathrm{mg} \mathrm{dm}^{-3}$} & \multicolumn{6}{c}{$\mathrm{cmolc} \mathrm{dm}^{-3}$} & & $\mathrm{~g} \mathrm{~kg}^{-1}$ & \\
\hline $0-10$ & 5.3 & 0.17 & 11.5 & 4 & 32 & 14 & 2.5 & 0.5 & 0.1 & 5.8 & 544 & 335 & 62 & 60 \\
$10-20$ & 4.8 & 0.14 & 10.4 & 2 & 22 & 12 & 0.8 & 0.4 & 0.6 & 6.6 & 447 & 396 & 57 & 100 \\
$20-30$ & 4.7 & 0.14 & 9.6 & 1 & 28 & 10 & 0.6 & 0.4 & 0.8 & 7.1 & 429 & 383 & 88 & 100 \\
$30-50$ & 4.6 & 0.11 & 5.1 & 1 & 16 & 8 & 0.4 & 0.3 & 1.0 & 6.3 & 381 & 379 & 80 & 160 \\
\hline
\end{tabular}

\section{Análises químicas do material vegetal}

A determinação das concentrações de nitrogênio $(\mathrm{N})$, fósforo $(\mathrm{P})$, potássio $(\mathrm{K})$, cálcio (Ca) e magnésio $(\mathrm{Mg})$ foi realizada a partir de extrato obtido por digestão do material vegetal em sistema de $\mathrm{H}_{2} \mathrm{SO}_{4} / \mathrm{H}_{2} \mathrm{O}_{2}$ a $270^{\circ} \mathrm{C}$. As concentrações de $\mathrm{N}$ foram determinadas pelo método de Kjeldahl enquanto que as concentrações de $\mathrm{P}$ foram determinadas por colorimetria em espectrofotômetro e os demais teores de nutrientes como $\mathrm{K}, \mathrm{Ca}$ e $\mathrm{Mg}$, foram determinados por espectrometria de absorção atômica.

As estimativas dos estoques de nutrientes foram obtidas multiplicando-se a concentração dos nutrientes pela massa seca de cada compartimento.

\section{Análise estatística}

Foram testados os efeitos da adubação, das espécies e da interação adubação $\mathrm{x}$ espécies nas variáveis estudadas por meio da análise de variância de dois fatores, com o auxílio do programa estatístico SIGMA PLOT versão 11 (Systat Software, 2010).

Quando necessário, os dados foram transformados (logaritmo neperiano e raiz quadrada) para atender aos pressupostos de normalidade dos erros e homogeneidade das variâncias. Os resultados, entretanto, foram apresentados com média e erro padrão originais. Aplicou-se o teste Tukey a $5 \%$ de probabilidade, para a comparação de médias.

\section{RESULTADOS E DISCUSSÃO}

O crescimento das espécies em altura e diâmetro durante o experimento está apresentado na Figura 1. A espécie $S$. paniculatum mostrou tendência a ser responsiva ao tratamento adubado sendo observados maiores valores numéricos em altura, diâmetro do coleto e diâmetro a altura do peito (dap), enquanto que a espécie $I$. edulis no tratamento não adubado foram verificados os menores valores. A faixa de valores dos resultados em altura e dap, está de acordo com estudos realizados com as mesmas espécies na região de estudo (ALEGRE et al., 2005, BRIENZA, 1999, MELO, 2002, OLIVEIRA et al., 2006, TONINI et al., 2006). E respostas de crescimento em altura à adubação fosfatada também foi observada em outros estudos realizados na região (JOSLIN et al., 2011, MARTINOTTO, 2006, OLIVEIRA et al., 2006, QUEIROZ et al., 2007).

A análise de variância da biomassa, estoques e concentrações de nutrientes dos compartimentos folha, galho e tronco das espécies introduzidas no sistema não apresentaram efeito significativo de Espécie $\mathrm{x}$ adubação efeitos de tratamentos $\mathrm{e}$ espécies (Tabelas 2 e 3). Houve efeito da adubação fosfatada na massa seca e estoque de fósforo das folhas e galhos, no estoque de nitrogênio dos galhos e nos estoques de cálcio e magnésio das folhas, galhos e tronco (Tabela 2) e na concentração de $\mathrm{Ca}$ das folhas (Tabela 3). Enquanto que houve efeito de espécies sobre a massa seca, N, P, K, $\mathrm{Ca}$ e $\mathrm{Mg}$ (Tabela 2) e concentrações de $\mathrm{K}, \mathrm{Ca}$ e $\mathrm{Mg}$ nos compartimentos folha, galho e tronco (Tabela 3).

Segundo os resultados, houve limitação pela disponibilidade de $\mathrm{P}$ nos acúmulos de massa seca e $\mathrm{P}$ nas folhas, galhos e tronco, acúmulo de $\mathrm{N}$ nas folhas e no acúmulo de $\mathrm{Ca}$ e $\mathrm{Mg}$ nas folhas, galhos e tronco das leguminosas utilizadas no experimento (Tabelas 4, 5 e 6). O tratamento adubado acumulou 


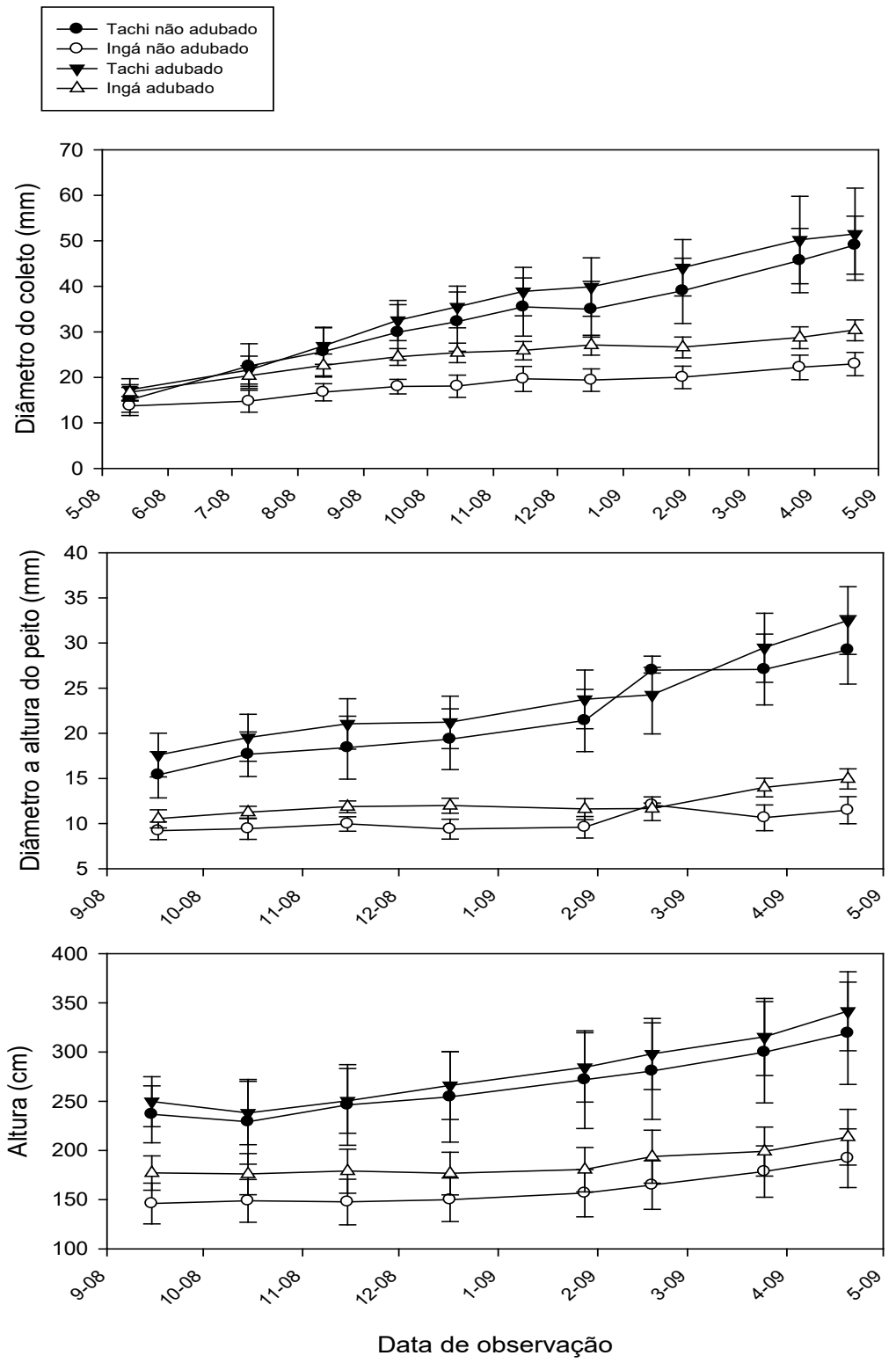

FIGURA 1: Médias mensais de diâmetro do coleto (A), diâmetro a altura do peito (B) e altura (C) das espécies Inga edulis e Sclerolobium paniculatum (Barras verticais representam \pm o erro padrão).

FIGURE 1: Monthly average of diameter (A), diameter at breast height (B) and height (C), and of Inga edulis and Sclerolobium paniculatum vertical bars represent \pm standard error).

aproximadamente duas vezes mais massa seca, $\mathrm{P}$, $\mathrm{Ca}$ e $\mathrm{Mg}$ nas folhas.

Não houve aumento das concentrações de nutrientes nas folhas, galhos e tronco das leguminosas com o tratamento de adubação, exceto para a concentração do cálcio foliar, (Tabela 7), provavelmente refletindo a composição química do fosfato natural de rocha de Arad - 33\% de P e 37\% de Ca (HOLLMMAN, 2004). A alta porosidade e a baixa dureza deste adubo propiciam liberação lenta e gradual de $\mathrm{Ca}$, aumentando a concentração deste elemento e diminuindo a fixação do $\mathrm{P}$ aos sesquióxidos de ferro e alumínio, podendo insolubilizar este elemento como fosfato de Cálcio tornando-o indisponível às plantas (GONÇALVES et al., 2008).

Em estudos realizados na região, observouse aumento nas concentrações de $\mathrm{P}$ foliar de espécies de vegetação secundária sob adubação fosfatada (DAVIDSON et al., 2004, GEHRING \& Vlek., 2004), diferentemente do que foi observado neste estudo. A concentração de nutriente nas 
TABELA 2: Análise de variância dos efeitos de tratamento (fosfato de Arad na dosagem 0 e $165 \mathrm{~kg}$ de $\mathrm{P}_{2} \mathrm{O}_{5}$ $\mathrm{ha}^{-1}$ ) e espécie (Sclerolobium paniculatum e Inga edulis) sobre a massa seca e estoques de nutrientes nos compartimentos folha, galho e tronco das leguminosas arbóreas utilizadas em plantio de enriquecimento de pousio em Marapanim - PA.

TABLE 2: Analysis of variance of treatment effects (Arad phosphate dosage 0 and $165 \mathrm{~kg}$ de $\mathrm{P}_{2} \mathrm{O}_{5}$ $\mathrm{ha}^{-1}$ ) and tree legume species (Sclerolobium paniculatum and Inga edulis) on dry matter and nutrient stocks in the leaves, branches and stem of tree legumes used in improvement of fallow, Marapanim, Pará state.

\begin{tabular}{cccccccc}
\hline \multirow{2}{*}{ Compartimento } & \multirow{2}{*}{ Fonte de variação } & Massa & \multicolumn{5}{c}{ Estoque } \\
\cline { 4 - 7 } & seca & Nitrogênio & Fósforo & Potássio & Cálcio & Magnésio \\
\hline \multirow{3}{*}{ Folha } & Tratamento & $9,18^{*}$ & 1,37 & $7,40^{*}$ & 0,69 & $15,99^{*}$ & $12,77^{*}$ \\
& Espécie & $92,18^{* * *}$ & $18,18^{*}$ & $55,38^{* *}$ & $40,52^{* *}$ & $37,28^{* *}$ & $8,64^{*}$ \\
& Tratamento x espécie & 1,78 & 0,92 & 2,53 & 0,04 & 2,70 & 0,66 \\
\hline \multirow{3}{*}{ Galho } & Tratamento & $7,38^{*}$ & $19,22^{*}$ & $9,94^{*}$ & 2,03 & 20,09 & $17,88^{*}$ \\
& Espécie & $59,62^{* *}$ & $64,16^{* *}$ & $53,53^{* *}$ & $32,19^{* *}$ & $45,21^{* *}$ & $47,14^{* *}$ \\
& Tratamento x espécie & 3,99 & 2,96 & 2,88 & 0,41 & 3,49 & 2,25 \\
\hline \multirow{3}{*}{ Tronco } & Tratamento & 4,86 & 4,44 & 5,02 & 0,02 & $5,63^{*}$ & $12,65^{*}$ \\
& Espécie & $51,67^{* *}$ & $44,02^{* *}$ & $34,88^{* *}$ & $15,33^{*}$ & $46,27^{* *}$ & $62,69^{* *}$ \\
& Tratamento x espécie & 2,16 & 1,04 & 1,33 & 0,81 & 1,00 & 1,21 \\
\hline
\end{tabular}

Em que: *, ** e ${ }^{* * *}$ Significativo a 5,1 e $0,1 \%$, respectivamente, pelo teste $\mathrm{F}$.

TABELA 3: Análise de variância dos efeitos de tratamento (fosfato de Arad na dosagem 0 e $165 \mathrm{~kg}$ de P2O5 ha-1) e espécie (Sclerolobium paniculatum e Inga edulis) sobre as concentrações de nutrientes nos compartimentos folha, galho e tronco das leguminosas arbóreas utilizadas em plantio de enriquecimento de pousio em Marapanim - PA.

TABLE 3: Analysis of variance of treatment effects (Arad phosphate dosage 0 and $165 \mathrm{~kg} \mathrm{de} \mathrm{P}_{2} \mathrm{O}_{5} \mathrm{ha}^{-1}$ ) and tree legume species (Sclerolobium paniculatum and Inga edulis) nutrient concentrations in the leaves, branches and stem of tree legumes used in improvement of fallow, Marapanim, Pará.

\begin{tabular}{|c|c|c|c|c|c|c|}
\hline \multirow{2}{*}{ Compartimento } & \multirow{2}{*}{$\begin{array}{l}\text { Fonte de } \\
\text { variação }\end{array}$} & \multicolumn{5}{|c|}{ Concentração } \\
\hline & & Nitrogênio & Fósforo & Potássio & Cálcio & Magnésio \\
\hline \multirow{3}{*}{ Folha } & Tratamento & 1,23 & 0,40 & 2,93 & $6,15^{*}$ & 1,12 \\
\hline & Espécie & 0,03 & 1,45 & $6,72 *$ & $67,27 * *$ & $36,90 * *$ \\
\hline & $\begin{array}{l}\text { Tratamento } \\
\mathrm{x} \text { espécie }\end{array}$ & 1,34 & 0,17 & 0,11 & 0,06 & 0,99 \\
\hline \multirow{3}{*}{ Galho } & Tratamento & 0,70 & 0,60 & 5,45 & 1,42 & 0,51 \\
\hline & Espécie & 1,31 & 1,57 & $29,51 * *$ & $35,27 * *$ & $44,86^{* *}$ \\
\hline & $\begin{array}{c}\text { Tratamento } \\
\mathrm{x} \text { espécie }\end{array}$ & 0,39 & 0,01 & 0,01 & 0,99 & 0,04 \\
\hline \multirow{3}{*}{ Tronco } & Tratamento & 0,05 & 3,93 & 1,37 & 0,21 & 0,02 \\
\hline & Espécie & 0,41 & 3,34 & $22,34 * *$ & $\begin{array}{c}26,41 \\
* *\end{array}$ & $10,18^{*}$ \\
\hline & $\begin{array}{l}\text { Tratamento } \\
\text { x espécie }\end{array}$ & 1,80 & 0,00 & 1,68 & 0,62 & 0,83 \\
\hline
\end{tabular}

Em que: ${ }^{*}, * * \mathrm{e}^{* * *}$ Significativo a 5,1 e $0,1 \%$, respectivamente, pelo teste $\mathrm{F}$. 
TABELA 4: Biomassa $\left(\mathrm{Mg} \mathrm{ha}^{-1}\right)$ e estoque de nutrientes $\left(\mathrm{kg} \mathrm{ha}^{-1}\right)$ de folhas das espécies Inga edulis e Sclerolobium paniculatum sob tratamentos adubado e não adubado, em Marapanim - PA (erro padrão entre parênteses, Letras maiúsculas indicam diferenças significativas entre espécies e letras minúsculas indicam diferença significativa entre tratamentos em nível de significância de $5 \%$ ).

TABLE 4: Leaf biomass $\left(\mathrm{Mg} \mathrm{ha}^{-1}\right)$ and nutrient stocks $\left(\mathrm{kg} \mathrm{ha}^{-1}\right)$ of Inga edulis e Sclerolobium paniculatum under fertilization and non-fertilization treatmentes in Marapanim, PA state (standard error in parentheses. Capital letters show significant differences between species and the lowercase ones indicate significant differences between treatments at a significance level of $5 \%$ ).

\begin{tabular}{|c|c|c|c|c|}
\hline \multirow{2}{*}{\multicolumn{2}{|c|}{ Tratamentos }} & \multicolumn{3}{|c|}{ Espécies } \\
\hline & & Sclerolobium paniculatum & Inga edulis & Média \\
\hline \multirow{3}{*}{$\begin{array}{l}\text { Massa Seca } \\
--\left(\mathrm{Mg} \mathrm{ha}^{-1}\right)--\end{array}$} & Não adubado & $2,27(0,4)$ & $0,25(0,1)$ & $1,26(0,4) b$ \\
\hline & Adubado & $3,34(0,4)$ & $0,67(0,2)$ & $2,01(0,6) \mathrm{a}$ \\
\hline & Média & $2,81(0,3) \mathrm{A}$ & $0,46(0,1) \mathrm{B}$ & \\
\hline \multirow{3}{*}{$\begin{array}{l}\text { Nitrogênio } \\
--\left(\mathrm{kg} \mathrm{ha}^{-1}\right)--\end{array}$} & Não adubado & $24,86(4,5)$ & $3,72(2,1)$ & $14,29(4,6)$ \\
\hline & Adubado & $38,50(12,7)$ & $5,05(0,4)$ & $21,77(8,6)$ \\
\hline & Média & $31,68(6,7) \mathrm{A}$ & $4,38(1,0) \mathrm{B}$ & \\
\hline \multirow{3}{*}{$\begin{array}{c}\text { Fósforo } \\
--\left(\mathrm{kg} \mathrm{ha}^{-1}\right)--\end{array}$} & Não adubado & $1,71(0,5)$ & $0,27(0,1)$ & $0,99(0,4) b$ \\
\hline & Adubado & $2,72(0,3)$ & $0,54(0,1)$ & $1,63(0,4) \mathrm{a}$ \\
\hline & Média & $2,21(0,3) \mathrm{A}$ & $0,41(0,1) \mathrm{B}$ & \\
\hline \multirow{3}{*}{$\begin{array}{l}\text { Potássio } \\
--\left(\mathrm{kg} \mathrm{ha}^{-1}\right)--\end{array}$} & Não adubado & $8,86(1,9)$ & $1,24(0,4)$ & $5,05(1,7)$ \\
\hline & Adubado & $9,58(1,3)$ & $2,48(0,5)$ & $6,03(1,5)$ \\
\hline & Média & $9,22(1,1) \mathrm{A}$ & $1,86(0,4) \mathrm{B}$ & \\
\hline \multirow{3}{*}{$\begin{array}{c}\text { Cálcio } \\
--\left(\mathrm{kg} \mathrm{ha}^{-1}\right)--\end{array}$} & Não adubado & $6,30(1,6)$ & $2,25(0,9)$ & $4,28(1,1) b$ \\
\hline & Adubado & $14,65(2,8)$ & $7,50(2,3)$ & $11,08(2,2) \mathrm{a}$ \\
\hline & Média & $10,48(2,2) \mathrm{A}$ & $4,88(1,5) \mathrm{B}$ & \\
\hline \multirow{3}{*}{$\begin{array}{l}\text { Magnésio } \\
--\left(\mathrm{kg} \mathrm{ha}^{-1}\right)--\end{array}$} & Não adubado & $2,39(0,5)$ & $0,59(0,2)$ & $1,49(0,4) b$ \\
\hline & Adubado & $4,64(0,6)$ & $1,56(0,4)$ & $3,10(0,7) \mathrm{a}$ \\
\hline & Média & $3,51(0,6) \mathrm{A}$ & $1,07(0,3) \mathrm{B}$ & \\
\hline
\end{tabular}

diferentes partes da planta é definida por dois processos dinâmicos, a absorção e transporte dos nutrientes e o acúmulo de matéria seca. $\mathrm{O}$ fato da adubação fosfatada ter afetado apenas o acúmulo de massa e estoques nutrientes e pouco efeito nas concentrações de nutrientes pode estar relacionado a estratégias de acúmulo de biomassa. Este fenômeno pode ser explicado pelo "efeito diluição" (JARREL et al., 1981), que geralmente é causado por um rápido aumento de matéria seca com o acúmulo de carboidratos e outras estruturas sólidas na planta, promovidos pela adubação, mas por algum motivo (insolubilização ou fixação do $\mathrm{P}$ no solo por exemplo), não acompanha um "consumo de luxo" ao ponto de aumentar as concentrações do elemento na planta.

O objetivo de adubar as leguminosas para aumentar a biomassa e estoques de nutrientes durante o período de pousio foi alcançado, conforme os resultados deste estudo. Dessa forma, é esperado aumento da ciclagem de nutrientes, neste sistema, podendo influenciar positivamente na produtividade da fase de cultivo e promover a sustentabilidade do sistema como um todo.

Em relação às espécies, Sclerolobium paniculatum foi a que apresentou maiores de massa seca e estoques de $\mathrm{N}, \mathrm{P}, \mathrm{K}, \mathrm{Ca}$ e $\mathrm{Mg}$ nos compartimentos folha, galho e tronco (Tabela 6), chegando a acumular de 6 a 8 vezes mais massa seca e 4 a 7 vezes mais $\mathrm{N}, \mathrm{P}, \mathrm{Ca}$ e $\mathrm{Mg}$, nos 
TABELA 5: Biomassa $\left(\mathrm{Mg} \mathrm{ha}^{-1}\right)$ e estoque de nutrientes $\left(\mathrm{kg} \mathrm{ha}^{-1}\right)$ de galhos das espécies Inga edulis e Sclerolobium paniculatum sob tratamentos adubado e não adubado, em Marapanim - PA (erro padrão entre parênteses, Letras maiúsculas indicam diferenças significativas entre espécies e letras minúsculas indicam diferença significativa entre tratamentos em nível de significância de $5 \%$ ).

TABLE 5: Branch biomass $\left(\mathrm{Mg} \mathrm{ha}^{-1}\right)$ and nutrient stocks $\left(\mathrm{kg} \mathrm{ha}^{-1}\right)$ of Inga edulis e Sclerolobium paniculatum under management of fertilization and non-fertilization in Marapanim, PA state (standard error in parentheses. Capital letters show significant differences between species and the lowercase ones indicate significant differences between treatments at a significance level of $5 \%$ ).

\begin{tabular}{|c|c|c|c|c|}
\hline \multirow{2}{*}{\multicolumn{2}{|c|}{ Tratamentos }} & \multicolumn{3}{|c|}{ Espécies } \\
\hline & & \multirow{2}{*}{$\frac{\text { Sclerolobium paniculatum }}{0,66(0,2)}$} & \multirow{2}{*}{$\begin{array}{l}\text { Inga edulis } \\
0,12(0,04)\end{array}$} & \multirow{2}{*}{$\frac{\text { Média }}{0,39(0,1) \mathrm{b}}$} \\
\hline Massa Seca & Não adubado & & & \\
\hline \multirow[t]{2}{*}{--(Mg ha-1)-- } & Adubado & $1,10(0,2)$ & $0,19(0,05)$ & $0,64(0,2) \mathrm{a}$ \\
\hline & Média & $0,88(0,1) \mathrm{A}$ & $0,15(0,03) \mathrm{B}$ & \\
\hline Nitrogênio & Não adubado & $5,36(1,2)$ & $1,12(0,42)$ & $3,24(1,0) b$ \\
\hline \multirow[t]{2}{*}{--(kg ha $\left.{ }^{-1}\right)--$} & Adubado & $9,48(0,8)$ & $2,89(1,61)$ & $6,19(1,5) \mathrm{a}$ \\
\hline & Média & $7,42(1,0) \mathrm{A}$ & $2,01(0,84) \mathrm{B}$ & \\
\hline Fósforo & Não adubado & $0,35(0,10)$ & $0,07(0,03)$ & $0,21(0,1) b$ \\
\hline \multirow[t]{2}{*}{$--\left(\mathrm{kg} \mathrm{ha}^{-1}\right)--$} & Adubado & $0,58(0,08)$ & $0,10(0,03)$ & $0,34(0,1) \mathrm{a}$ \\
\hline & Média & $0,46(0,07) \mathrm{A}$ & $0,08(0,02) \mathrm{B}$ & \\
\hline Potássio & Não adubado & $2,75(0,8)$ & $0,69(0,26)$ & $1,72(0,5)$ \\
\hline \multirow[t]{2}{*}{$--\left(\mathrm{kg} \mathrm{ha}^{-1}\right)--$} & Adubado & $3,61(0,6)$ & $0,97(0,30)$ & $2,29(0,6)$ \\
\hline & Média & $3,18(0,5) \mathrm{A}$ & $0,83(0,19) \mathrm{B}$ & \\
\hline Cálcio & Não adubado & $2,25(0,5)$ & $0,75(0,30)$ & $1,50(0,4) b$ \\
\hline \multirow[t]{2}{*}{--(kg ha-1)-- } & Adubado & $3,78(0,5)$ & $1,51(0,51)$ & $2,65(0,5) \mathrm{a}$ \\
\hline & Média & $3,02(0,4) \mathrm{A}$ & $1,13(0,31) \mathrm{B}$ & \\
\hline Magnésio & Não adubado & $0,49(0,1)$ & $0,18(0,06)$ & $0,33(0,1) b$ \\
\hline \multirow[t]{2}{*}{$--\left(\mathrm{kg} \mathrm{ha}^{-1}\right)--$} & Adubado & $0,92(0,1)$ & $0,31(0,08)$ & $0,62(0,1) \mathrm{a}$ \\
\hline & Média & $0,70(0,1) \mathrm{A}$ & $0,25(0,05) \mathrm{B}$ & \\
\hline
\end{tabular}

compartimentos folha, galho e tronco que a espécie Inga edulis (Tabelas 4, 5 e 6). Por outro lado, I. edulis apresentou teores de $\mathrm{K}, \mathrm{Ca}$ e $\mathrm{Mg}$ significativamente maiores, nos compartimentos folha, galho e tronco (Tabelas 7, 8 e 9). Enquanto Inga edulis apresentou maiores teores, para Sclerolobium paniculatum foram quantificados maiores valores de estoques desses nutrientes.

A faixa de resultados das concentrações de nutrientes encontradas neste estudo é comparável aos verificados na literatura para espécies leguminosas arbóreas (QUEIROZ et al., 2007). Os elevados teores de $\mathrm{K}, \mathrm{Ca}$ e $\mathrm{Mg}$ encontrados na espécie Inga edulis neste estudo podem ser considerados como característica própria da espécie. Há relatos na literatura de que a espécie apresenta maiores concentrações de nutrientes em relação à Sclerolobium paniculatum. Santos Jr. et al. (2006) em estudo de plantio de Inga edulis em área degradada na Amazônia, encontraram teores de N, P, K, Ca e Mg iguais a 29,27, 1,04, 12,59 e 2,05, respectivamente. Enquanto que Medeiros e Haridason (1995) em estudo em plantio de Sclerolobium paniculatum no cerrado verificaram concentrações de N, P, K, Ca e Mg iguais a 16,3, $0,6,3,3,1,4$, e 0,7 respectivamente.

\section{CONCLUSÕES}

O acúmulo de massa seca e nutrientes como 
TABELA 6: Biomassa $\left(\mathrm{Mg} \mathrm{ha}^{-1}\right)$ e estoque de nutrientes $\left(\mathrm{kg} \mathrm{ha}^{-1}\right)$ de tronco das espécies Inga edulis e Sclerolobium paniculatum sob tratamentos adubado e não adubado, em Marapanim - PA (erro padrão entre parênteses Letras maiúsculas indicam diferenças significativas entre espécies e letras minúsculas indicam diferença significativa entre tratamentos ao nível de significância de $5 \%$ ).

TABLE 6: Stem, biomass $\left(\mathrm{Mg} \mathrm{ha}^{-1}\right)$ and nutrient stocks $\left(\mathrm{kg} \mathrm{ha}^{-1}\right)$ of stem of Inga edulis e Sclerolobium paniculatum under management of fertilization and non-fertilization in Marapanim, PA state (standard error in parentheses. Capital letters show significant differences between species and the lowercase ones indicate significant differences between treatments at a significance level of $5 \%)$.

\begin{tabular}{|c|c|c|c|c|}
\hline \multirow{2}{*}{\multicolumn{2}{|c|}{ Tratamentos }} & \multicolumn{3}{|c|}{ TRONCO } \\
\hline & & \multirow{2}{*}{$\frac{\text { Sclerolobium paniculatum }}{2,62(0,6)}$} & \multirow{2}{*}{$\begin{array}{c}\text { Inga edulis } \\
0,29(0,08)\end{array}$} & \multirow{2}{*}{$\begin{array}{c}\text { Média } \\
1,46(0,5) \mathrm{b}\end{array}$} \\
\hline Massa Seca & Não adubado & & & \\
\hline \multirow[t]{2}{*}{--(Mg ha-1)-- } & Adubado & $4,12(0,7)$ & $0,59(0,12)$ & $2,36(0,7) \mathrm{a}$ \\
\hline & Média & $3,37(0,5) \mathrm{A}$ & $0,44(0,09) \mathrm{B}$ & \\
\hline Nitrogênio & Não adubado & $20,97(4,4)$ & $2,23(0,45)$ & $11,60(4,1)$ \\
\hline \multirow[t]{2}{*}{$--\left(\mathrm{kg} \mathrm{ha}^{-1}\right)--$} & Adubado & $31,44(4,5)$ & $5,85(1,42)$ & $18,64(5,3)$ \\
\hline & Média & $26,20(3,5) \mathrm{A}$ & $4,04(0,97) \mathrm{B}$ & \\
\hline Fósforo & Não adubado & $0,98(0,3)$ & $0,11(0,02)$ & $0,55(0,2)$ \\
\hline \multirow[t]{2}{*}{--(kg ha-1)-- } & Adubado & $1,64(0,3)$ & $0,29(0,09)$ & $0,96(0,3)$ \\
\hline & Média & $1,31(0,2) \mathrm{A}$ & $0,20(0,05) \mathrm{B}$ & \\
\hline Potássio & Não adubado & $7,92(3,1)$ & $1,35(0,44)$ & $4,63(1,9)$ \\
\hline \multirow[t]{2}{*}{$--\left(\mathrm{kg} \mathrm{ha}^{-1}\right)--$} & Adubado & $6,89(1,1)$ & $2,81(0,87)$ & $4,85(1,0)$ \\
\hline & Média & $7,41(1,5) \mathrm{A}$ & $2,08(0,43) \mathrm{B}$ & \\
\hline Cálcio & Não adubado & $5,02(1,1)$ & $0,99(0,30)$ & $3,01(0,9) \mathrm{b}$ \\
\hline \multirow[t]{2}{*}{--( $\left(\mathrm{kg} \mathrm{ha}^{-1}\right)--$} & Adubado & $7,80(1,6)$ & $2,44(1,01)$ & $5,12(1,4) \mathrm{a}$ \\
\hline & Média & $6,41(1,1) \mathrm{A}$ & $1,72(0,53) \mathrm{B}$ & \\
\hline Magnésio & Não adubado & $1,56(0,4)$ & $0,21(0,04)$ & $0,88(0,3) b$ \\
\hline \multirow[t]{2}{*}{$--\left(\mathrm{kg} \mathrm{ha}^{-1}\right)--$} & Adubado & $2,36(0,5)$ & $0,53(0,19)$ & $1,44(0,4) \mathrm{a}$ \\
\hline & Média & $1,96(0,3) \mathrm{A}$ & $0,37(0,11) \mathrm{B}$ & \\
\hline
\end{tabular}

$\mathrm{N}, \mathrm{P}, \mathrm{Ca}$ e Mg das leguminosas arbóreas utilizadas no melhoramento de pousio (Sclerolobium paniculatum e Inga edulis) é limitado pela disponibilidade de fósforo no solo.

Sclerobium paniculatum é mais eficiente que Inga edulis quanto ao acúmulo de biomassa e nutrientes, como nitrogênio, fósforo, cálcio e magnésio sob as condições experimentais do sistema de pousio deste estudo, tanto com adubação fosfatada ou não. Entretanto, a adubação fosfatada, como a empregada, afetou positivamente a concentração de cálcio nas folhas dessas espécies.

A adubação fosfatada de baixa solubilidade na dosagem de $200 \mathrm{~g}$ por cova $\left(165 \mathrm{~kg}\right.$ de $\mathrm{P}_{2} \mathrm{O}_{5}$ ha $^{-1}$ ) afeta positivamente a concentração de cálcio nas folhas das leguminosas utilizadas neste experimento.

\section{AGRADECIMENTOS}

Ao Programa Nacional de Pós-Doutorado institucional da Capes (Auxilio 2405/2011) pela concessão de bolsa ao primeiro autor e à Embrapa Amazônia Oriental e Universidade Federal Rural da Amazônia pelo apoio logístico e financeiro no desenvolvimento do experimento. Aos engenheiros florestais Aline F Paim, Kelen P Soares e Luiz Thiago B Greff pela colaboração na coleta de biomassa das árvores. 
TABELA 7: Concentração de nutrientes $\left(\mathrm{g} \mathrm{kg}^{-1}\right)$ em folhas das espécies Inga edulis e Sclerolobium paniculatum sob tratamentos adubado e não adubado, em Marapanim - PA (erro padrão entre parênteses, Letras maiúsculas indicam diferenças significativas entre espécies e letras minúsculas indicam diferença significativa entre tratamentos em nível de significância de 5\%).

TABLE 7: Nutrient concentrations $\left(\mathrm{g} \mathrm{kg}^{-1}\right)$ in leaves of Inga edulis and Sclerolobium paniculatum under management of fertilization and non-fertilization in Marapanim, PA state (standard error in parentheses Capital letter show significant differences between species and lowercase indicate significant differences between treatments at a significance level of 5\%).

\begin{tabular}{ccccc}
\hline Concentração de Nutrientes & \multirow{2}{*}{ Tratamentos } & \multicolumn{3}{c}{ Espécies } \\
\cline { 3 - 5 } \cline { 3 - 5 } ---(g.kg-1)--- & & Sclerolobium paniculatum & Inga edulis & Média \\
\hline \multirow{3}{*}{ Nitrogênio } & Não adubado & $11,2(1,34)$ & $13,1(2,75)$ & $12,1(1,5)$ \\
& Adubado & $11,3(2,64)$ & $8,7(1,66)$ & $10,0(1,5)$ \\
& Média & $11,2(1,37)$ & $10,9(1,70)$ & \\
\hline \multirow{2}{*}{ Fósforo } & Não adubado & $0,7(0,12)$ & $0,9(0,21)$ & $0,8(0,1)$ \\
& Adubado & $0,8(0,03)$ & $0,9(0,17)$ & $0,9(0,1)$ \\
& Média & $0,8(0,06)$ & $0,9(0,13)$ & \\
\hline \multirow{2}{*}{ Potássio } & Não adubado & $3,8(0,30)$ & $5,6(0,70)$ & $4,7(0,5)$ \\
& Adubado & $2,9(0,20)$ & $4,3(0,88)$ & $3,6(0,5)$ \\
& Média & $3,3(0,23) \mathrm{B}$ & $4,9(0,57) \mathrm{A}$ & \\
\hline \multirow{2}{*}{ Cálcio } & Não adubado & $2,6(0,40)$ & $8,9(0,33)$ & $5,8(1,2) \mathrm{b}$ \\
& Adubado & $4,4(0,66)$ & $11,1(0,88)$ & $7,7(1,4) \mathrm{a}$ \\
& Média & $3,5(0,49) \mathrm{B}$ & $10,0(0,60) \mathrm{A}$ & \\
\hline \multirow{2}{*}{ Magnésio } & Não adubado & $1,0(0,11)$ & $2,4(0,11)$ & $1,7(0,3)$ \\
& Adubado & $1,4(0,11)$ & $2,4(0,24)$ & $1,9(0,2)$ \\
& Média & $1,2(0,10) \mathrm{B}$ & $2,4(0,12) \mathrm{A}$ & \\
\hline
\end{tabular}

TABELA 8: Concentração de nutrientes $\left(\mathrm{g} \mathrm{kg}^{-1}\right)$ em galhos das espécies Inga edulis e Sclerolobium paniculatum sob tratamentos adubado e não adubado, em Marapanim - PA (erro padrão entre parênteses Letras maiúsculas indicam diferenças significativas entre espécies e letras minúsculas indicam diferença significativa entre tratamentos ao nível de significância de 5\%).

TABLE 8: Nutrient concentrations $\left(\mathrm{g} \mathrm{kg}^{-1}\right)$ in brunches of Inga edulis and Sclerolobium paniculatum under management of fertilization and non-fertilization in Marapanim, PA state (standard error in parentheses. Capital letters show significant differences between species and lowercase indicate significant differences between treatments at a significance level of 5\%).

\begin{tabular}{ccccc}
\hline \multirow{2}{*}{ Nutrientes } & Tratamentos & \multicolumn{3}{c}{ Espécies } \\
\cline { 3 - 5 } & & Sclerolobium paniculatum & Inga edulis & Média \\
\hline \multirow{3}{*}{ Nitrogênio } & Não adubado & $8,4(0,61)$ & $9,8(1,00)$ & $9,1(0,60)$ \\
& Adubado & $9,0(0,89)$ & $13,8(4,90)$ & $11,4(2,48)$ \\
& Média & $8,7(0,51)$ & $11,8(2,43)$ & \\
\hline \multirow{3}{*}{ Fósforo } & Não adubado & $0,5(0,05)$ & $0,6(0,04)$ & $0,5(0,03)$ \\
& Adubado & $0,5(0,03)$ & $0,6(0,04)$ & $0,6(0,03)$ \\
& Média & $0,5(0,03)$ & $0,6(0,03)$ & \\
\hline
\end{tabular}


TABELA 8: Continuação...

TABLE 8: Continued...

\begin{tabular}{ccccc}
\hline \multirow{2}{*}{ Nutrientes } & Tratamentos & \multicolumn{3}{c}{ Espécies } \\
\cline { 3 - 5 } & & Sclerolobium paniculatum & Inga edulis & Média \\
\hline \hline \multirow{3}{*}{ Potássio } & Não adubado & $4,0(0,27)$ & $5,8(0,38)$ & $4,9(0,40)$ \\
& Adubado & $3,3(0,37)$ & $5,0(0,52)$ & $4,2(0,44)$ \\
& Média & $3,7(0,25) \mathrm{B}$ & $5,4(0,33) \mathrm{A}$ & \\
\hline \multirow{3}{*}{ Cálcio } & Não adubado & $3,4(0,30)$ & $6,6(0,68)$ & $5,0(0,69)$ \\
& Adubado & $3,5(0,22)$ & $8,0(1,01)$ & $5,7(0,97)$ \\
& Média & $3,5(0,17) \mathrm{B}$ & $7,3(0,62) \mathrm{A}$ & \\
\hline \multirow{3}{*}{ Magnésio } & Não adubado & $0,7(0,06)$ & $1,7(0,18)$ & $1,2(0,19)$ \\
& Adubado & $0,9(0,13)$ & $1,7(0,13)$ & $1,3(0,18)$ \\
& Média & $0,8(0,07) \mathrm{B}$ & $1,7(0,10) \mathrm{A}$ & \\
\hline
\end{tabular}

TABELA 9: Concentração de nutrientes $\left(\mathrm{g} \mathrm{kg}^{-1}\right)$ em tronco das espécies Inga edulis e Sclerolobium paniculatum sob tratamentos adubado e não adubado, em Marapanim - PA (erro padrão entre parênteses, Letras maiúsculas indicam diferenças significativas entre espécies e letras minúsculas indicam diferença significativa entre tratamentos em nível de significância de 5\%).

TABLE 9: Nutrient concentrations $\left(\mathrm{g} \mathrm{kg}^{-1}\right)$ in stems of Inga edulis and Sclerolobium paniculatum under management of fertilization and non-fertilization in Marapanim, PA state (standard error in parentheses. Capital letters (uppercase letters) show significant differences between species and lowercase letters indicate significant differences between treatments at a significance level of $5 \%$ ).

\begin{tabular}{ccccr}
\hline \multirow{2}{*}{ Nutrientes } & Tratamentos & \multicolumn{3}{c}{ Espécies } \\
\cline { 3 - 5 } & & Sclerolobium paniculatum & Inga edulis & Média \\
\hline \multirow{3}{*}{ Nitrogênio } & Não adubado & $9,0(1,70)$ & $8,4(1,78)$ & $8,7(1,15)$ \\
& Adubado & $8,0(1,38)$ & $9,8(1,41)$ & $8,9(0,97)$ \\
& Média & $8,5(1,03)$ & $9,1(1,08)$ & \\
\hline \multirow{3}{*}{ Fósforo } & Não adubado & $0,3(0,06)$ & $0,4(0,03)$ & $0,4(0,03)$ \\
& Adubado & $0,4(0,01)$ & $0,5(0,04)$ & $0,4(0,02)$ \\
& Média & $0,4(0,03)$ & $0,4(0,03)$ & \\
\hline \multirow{3}{*}{ Potássio } & Não adubado & $2,8(0,83)$ & $4,4(0,53)$ & $3,6(0,55)$ \\
& Adubado & $1,7(0,11)$ & $4,5(0,38)$ & $3,1(0,56)$ \\
& Média & $2,3(0,45) \mathrm{B}$ & $4,5(0,30) \mathrm{A}$ & \\
\hline \multirow{3}{*}{ Cálcio } & Não adubado & $2,0(0,10)$ & $3,3(0,20)$ & $2,6(0,27)$ \\
& Adubado & $1,9(0,12)$ & $3,7(0,66)$ & $2,8(0,46)$ \\
& Média & $1,9(0,07) \mathrm{B}$ & $3,5(0,33) \mathrm{A}$ & \\
\hline \multirow{3}{*}{ Magnésio } & Não adubado & $0,6(0,06)$ & $0,8(0,09)$ & $0,7(0,06)$ \\
& Adubado & $0,6(0,05)$ & $0,8(0,13)$ & $0,7(0,08)$ \\
& Média & $0,6(0,04) \mathrm{B}$ & $0,8(0,07) \mathrm{A}$ & \\
\hline
\end{tabular}




\section{REFERÊNCIAS BIBLIOGRÁFICAS}

ALEGRE, J. C. et al. Planted tree fallows for improving land productivity in the humid tropics of Peru. Agriculture Ecosystems \& Environment, v.110, n.1-2, p.104-117, 2005.

ALMEIDA, E., SABOGAL, C.; BRIENZA JR, S. Recuperação de áreas alteradas na Amazônia brasileira: Experiências locais, lições aprendidas e implicações para políticas públicas. Bogor: CIFOR. 2006. 202p.

BARRIOS, E.; COBO, J. G. Plant growth, biomass production and nutrient accumulation by slash/ mulch agroforestry systems in tropical hillsides of Colombia. Agroforestry Systems, v.60, n.3, p.255-265, 2004.

BASAMBA, T. A. et al. Impact of planted fallows and a crop rotation on nitrogen mineralization and phosphorus and organic matter fractions on a Colombian volcanic-ash soil. Nutrient Cycling in Agroecosystems, v.77, n.2, p.127-141, 2007.

BRIENZA JÚNIOR, S. Enriquecimento de Florestas secundárias como tecnologia de produção sustentável para a agricultura familiar. Ciências Naturais, v.7, n.3, p.331-337, 2012.

BRIENZA Júnior, S. Biomass dynamics of fallow vegetation enriched with leguminous trees in the Eastern Amazon of Brazil. PhD. Gottingen University. Gottingen. 1999. 133p.

DAVIDSON, E. A. et al. Nitrogen and phosphorus limitation of biomass growth in a tropical secondary forest. Ecological Applications, v.14, n.4, p.S150-S163, 2004.

DENICH, M. et al. A concept for the development of fire-free fallow management in the Eastern Amazon, Brazil. Agriculture Ecosystems \& Environment, v.110, n.1-2, p.43-58, 2005.

GEHRING, C.; VLEK, P. L. G. Limitations of the $\mathrm{N}-15$ natural abundance method for estimating biological nitrogen fixation in Amazonian forest legumes. Basic and Applied Ecology, v.5, n.6, p.567-580, 2004.

GONÇALVES, G. K. et al. Solubilização dos fosfatos naturais Patos de Minas e Arad em dois solos alagados. Revista Brasileira de Ciência do Solo, v.32, p.2157-2164, 2008.

GÜSEWELL, S. N:P ratios in terrestrial plants: variation and functional significance. New Phytologist, v.164, p.243-266, 2004.

JOSLIN, A et al. Five native tree species and manioc under slash-and-mulch agroforestry in the eastern
Amazon of Brazil: plant growth and soil responses. Agroforestry Systems, v.81, n.1, p.1-14, 2011.

KOERSELMAN, W.; MEULEMAN, A. F. M. The vegetation $\mathrm{N}: \mathrm{P}$ ratio: a new tool to detect the nature of nutrient limitation. Journal Applied Ecology, v.33, p.1441-1450, 1996.

LAWRENCE, D. Nitrogen and phosphorus enhance growth and luxury consumption of four secondary forest tree species in Borneo. Journal of Tropical Ecology, v.17, p.859-869, 2001.

MARTINOTTO, F. Avaliação do desenvolvimento inicial de espécies arbóreas nativas do cerrado. Especialização em Agricultura Tropical. Universidade Federal do Mato grosso. Cuiabá. 2006. 60p.

MCGRATH, D. A. et al. Soil phosphorus availability and fine root proliferation in Amazonian agroforests 6 years following forest conversion. Agriculture, Ecosystems \& Environment, v.83, n.3, p.271-284, 2001.

MEDEIROS, R. A.; HARIDASON, M. Seasonal Variations in the foliar concentrations of nutrients in some aluminium accumulating and non-accumulating species of the cerrado region of central Brazil. Plant and Soil, v.88, p.433-436, 1995.

QUEIROZ, L. R. et al. Avaliação da produtividade de fitomassa e acúmulo de $\mathrm{N} \mathrm{P}$ e $\mathrm{K}$ em leguminosas arbóreas no sistema em aléias, em Campos dos Goytacazes, RJ. Revista Árvore, v.31, p.383-390, 2007.

SANCHEZ, P. A. Improved fallows come of age in the tropics. Agroforestry Systems, v.47, n.1-3, p.3-12, 1999.

SANTOS JR, U. M. et al. Growth, leaf nutrient concentration and photosynthetic nutrient use efficiency in tropical tree species planted in degraded areas in central Amazonia. Forest Ecology and Management, v.226, n.1-3, p.299-309, 2006.

SOMMER, R. Water and nutrient balance in deep soils under shifting cultivation with and without burning in the Eastern Amazon. Gottingen Universit. Gottingen. 2000. 240p.

TONINI, H. et al. Avaliação de Especies Florestais em Área de Mata no Estado de Roraima. Cerne, v.12, n.1, p. 8-18, 2006.

ZARIN, D. J. et al. Legacy of fire slows carbon accumulation in Amazonian forest regrowth. Frontiers in Ecology and the Environment, v.3, n.7, p.365-369, 2005. 